\title{
Efficiency of Prediction Error and Instrumental Variable Methods for Closed-loop Identification
}

\author{
U. Forssell and C.T. Chou \\ Department of Electrical Engineering \\ Linkping University, S-581 83 Linkping, Sweden \\ WWW: http://www.control.isy.liu.se \\ Email: ufo@isy.liu.se, chou@harding.et.tudelft.nl
}

February 26, 1998

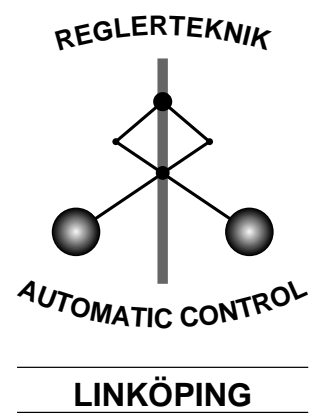

Report no.: LiTH-ISY-R-2015

Submitted to CDC '98

Technical reports from the Automatic Control group in Linkping are available by anonymous ftp at the address ftp.control.isy.liu.se. This report is contained in the compressed postscript file 2015.ps.z. 


\title{
Efficiency of Prediction Error and Instrumental Variable Methods for Closed-loop Identification
}

\author{
Urban Forssell* and C.T. Chou ${ }^{\dagger}$
}

February 26, 1998

\begin{abstract}
We study the efficiency of a number of closed-loop identification methods. Results will be given for methods based on the prediction error approach as well as those based on the instrumental variable approach. It is shown that all methods typically gives worse accuracy than a directly applied prediction error method. The key to this result is that in the direct method all the input signal power is utilized in reducing the variance while in the other methods only certain parts of the input spectrum is used, thus reducing the signal-to-noise ratio and consequently increasing the variance. Conditions for the instrumental variable method to give optimal accuracy are also given. Moreover, interesting insights in the properties of a recently suggested subspace method for closed-loop identification are obtained by exploring the links between this method and the instrumental variable method.
\end{abstract}

\section{Introduction}

Identification of systems operating under output feedback (i.e., in closed-loop) has been the topic of many papers since the sixties. Initially the focus was on identifiability and accuracy issues [3]. With the increased interest in model based control the focus shifted towards problems concerning bias error, due to under-modeling, and especially on how to affect this bias error in the identified models with pre-filters.

It has been known for some time that a directly applied prediction error (PE) method gives consistency as long as the true system (including the noise properties) can be correctly described within the model class [5]. In practice this calls for flexible parameterized noise models/pre-filters which implies that with this method the bias distribution cannot be manipulated at will. In order to circumvent the need for parameterized noise models, researchers have come up with alternative PE methods for closed-loop identification. We will study two of them here: the indirect method and the two-stage method [11]. These three methods will be introduced and studied in Section 3 . In the analysis we will exclusively discuss efficiency. It will be shown that the latter two methods give sub-optimal accuracy, unless the signals are noise free.

The instrumental variable (IV) methods [7] form a different class of identification methods that is related, but not equivalent, to the prediction error methods. IV variants for closed-loop identification have also been suggested and the statistical properties of these methods been analyzed. The efficiency of closed-loop IV methods has previously been studied in, e.g., [9] and we will re-use some results from there in this paper. Under certain circumstances the IV method can give the same level of accuracy as the direct PE method. In general though, the accuracy will be sub-optimal.

\footnotetext{
* Division of Automatic Control, Department of Electrical Engineering, Linköping University, S-581 83 Linköping, Sweden. Email: ufo@isy.liu.se. U. Forssell is supported by the Swedish Research Council for Engineering Sciences.

$\dagger^{\dagger}$ Department of Electrical Engineering, Delft University of Technology, P.O. Box 5031, 2600 GA Delft, The Netherlands. Email: chou@harding.et.tudelft.nl. C.T. Chou is supported by the Dutch Technology Foundation (STW) under project number DEL55.3891.
} 
Recently a new subspace method which can also be applied to closed-loop data was introduced in [1]. This scheme, which will referred to as the errors-in-variables (EIV) scheme as it solves an EIV problem, is based on IV and its efficiency can be studied in the same vein as that in [13] where the statistical properties of IV based subspace identification methods are analyzed. However, the resulting variance expression, which has the same form as that appeared in [13], does not provide a useful platform for comparison with other identification methods. In this paper, we have chosen to analyze a modified, but yet representative, form of the EIV algorithm based on single-input single-output (SISO) ARMAX models. This provides a useful platform for cross comparison as well as valuable insights into the properties of this algorithm.

The rest of this paper is organized as follows: Section 2 defines the model and various notation to be used in this paper. Sections 3 and 4 analyze respectively the efficiency of prediction error based and IV based closed-loop identification methods. Section 5 gives a discussion on the major results of this paper. Section 6 gives a simple but illustrative example and finally the conclusions are to be found in Section 7.

\section{Preliminaries}

We assume that the true system is given by

$$
A_{0}(q) y(t)=B_{0}(q) u(t)+C_{0}(q) e(t)
$$

where

$$
\begin{aligned}
& A_{0}(q)=1+a_{1,0} q^{-1}+\ldots+a_{n_{a}, 0} q^{-n_{a}} \\
& B_{0}(q)=q^{-n_{k}}\left(b_{0,0}+b_{1,0} q^{-1}+\ldots+b_{n_{b}, 0} q^{-n_{b}}\right) \\
& C_{0}(q)=1+c_{1,0} q^{-1}+\ldots+c_{n_{c}, 0} q^{-n_{c}}
\end{aligned}
$$

with $n_{a} \geq n_{b}, n_{c} ; n_{k} \geq 0$. The polynomial $C_{0}(q)$ is assumed to be Hurwitz, the noise $e(t)$ is white with variance $\lambda_{0}$. To identify this system we will work with models of the form

$$
\hat{y}(t \mid \theta)=\varphi^{T}(t) \theta
$$

where

$$
\begin{aligned}
\theta & =\left[a_{1}, \ldots, a_{n_{a}}, b_{0}, \ldots, b_{n_{b}}\right]^{T} \\
\varphi(t) & =\left[-y(t-1), \ldots,-y\left(t-n_{a}\right), u\left(t-n_{k}\right), \ldots, u\left(t-n_{k}-n_{b}\right)\right]^{T}
\end{aligned}
$$

The "true" parameter vector will be denoted by $\theta_{0}$. In some cases the model (5) will be applied together with a (possibly parameter-dependent) monic pre-filter $L$ that can be used to emphasize certain frequency regions. A standard result in identification is that the estimate $\hat{\theta}_{N}$, obtained using a prediction error method or instrumental variable method based on $N$ data samples, obeys

$$
\begin{aligned}
& \hat{\theta}_{N} \rightarrow \theta^{*}, \quad \text { with probability } 1 \text { as } N \rightarrow \infty \\
& \sqrt{N}\left(\hat{\theta}_{N}-\theta^{*}\right) \in \operatorname{AsN}\left(0, P_{\theta}\right)
\end{aligned}
$$

In this paper we will characterize the covariance matrix $P_{\theta}$ for a number of identification methods that guarantee $\theta^{*}=\theta_{0}$ (i.e. that the parameter estimates converge to the true values).

We assume that there is a stabilizing LTI feedback between $u(t)$ and $y(t)$ given by

$$
u(t)=r(t)-K(q) y(t)=r(t)-\frac{S(q)}{R(q)} y(t)
$$

with $r(t)$ being a "reference signal" and

$$
\begin{aligned}
& R(q)=1+r_{1} q^{-1}+\ldots+r_{n_{r}} q^{-n_{r}} \\
& S(q)=s_{0}+s_{1} q^{-1}+\ldots+s_{n_{s}} q^{-n_{s}}
\end{aligned}
$$


In addition the feedback loop is assumed to be well posed and $r(t)$ is assumed to be independent of $e(t)$.

By combining (1) and (10) it can be shown that the parts of the input and output that are due to the reference signal are

$$
\begin{aligned}
& u_{r}(t)=\frac{A_{0}(q) R(q)}{A_{0}(q) R(q)+B_{0}(q) S(q)} r(t) \\
& y_{r}(t)=\frac{B_{0}(q) R(q)}{A_{0}(q) R(q)+B_{0}(q) S(q)} r(t)=\frac{B_{0}(q)}{A_{0}(q)} u_{r}(t)
\end{aligned}
$$

Similarly one can define $u_{e}(t)$ and $y_{e}(t)$ as the parts of $u(t)$ and $y(t)$ that are due to the noise $e(t)$. We thus have $u(t)=u_{r}(t)+u_{e}(t)$ and $y(t)=y_{r}(t)+y_{e}(t)$. In the same vein the regression vector $\varphi(t)$ can be split up into two parts:

$$
\varphi(t)=\varphi_{r}(t)+\varphi_{e}(t)
$$

where $\varphi_{r}(t)$ and $\varphi_{e}(t)$ are the parts of $\varphi(t)$ that are due to $r(t)$ and $e(t)$, respectively.

\section{Prediction Error Methods}

\subsection{General}

Methods that aim at minimizing the prediction errors are perhaps the most common among all identification methods. With measured data $Z^{N}=\{y(1), u(1), \ldots, y(N), u(N)\}$ and a parameterized model structure $\hat{y}(t \mid \theta)$ the prediction error estimate is found as the straightforward fit [5]:

$$
\begin{aligned}
\hat{\theta}_{N} & =\arg \min _{\theta} \frac{1}{N} \sum_{t=1}^{N} \varepsilon_{F}^{2}(t, \theta) \\
\varepsilon_{F}(t, \theta) & =L(q, \theta)(y(t)-\hat{y}(t \mid \theta))
\end{aligned}
$$

Here $L(q, \theta)$ is a monic pre-filter. Alternatively $L(q, \theta)$ can be viewed as an inverse noise model used to whiten the prediction errors.

This approach can also be applied to closed-loop identification. Several different "methods" and parameterizations have been suggested. See, e.g., [12, 2]. Here we will discuss three of them, namely the so called direct, indirect, and two-stage methods.

\subsection{The Direct Method}

The most basic one is simply to ignore the feedback and apply the method directly to measured input-output data. This is called the direct method and coincides with the standard prediction error method for open-loop identification, albeit some care has to be exercised in the analysis in case of closed-loop data.

The direct method gives consistency and optimal accuracy provided that the parameterization is flexible enough. The requirement is that there exists a $\theta_{0}$ in the parameter set such that $\varepsilon\left(t, \theta_{0}\right)=e(t)$. Under fairly mild additional conditions we also have

$$
\begin{aligned}
\sqrt{N}\left(\hat{\theta}_{N}-\theta_{0}\right) & \in A s N\left(0, P_{\theta}\right) \\
P_{\theta} & =\lambda_{0}\left[\bar{E} \psi\left(t, \theta_{0}\right) \psi^{T}\left(t, \theta_{0}\right)\right]^{-1} \\
\psi\left(t, \theta_{0}\right) & =-\left.\frac{d}{d \theta} \varepsilon_{F}(t, \theta)\right|_{\theta=\theta_{0}}
\end{aligned}
$$

By the chain rule, the gradient vector $\psi(t, \theta)$ can be written (cf. (5) and (17))

$$
\psi(t, \theta)=L^{\prime}(q, \theta)\left(y(t)-\varphi^{T}(t) \theta\right)+L(q, \theta) \varphi(t)
$$


Since $L\left(q, \theta_{0}\right)=1 / C_{0}(q)$ and $y(t)-\varphi^{T}(t) \theta_{0}=C_{0}(q) e(t)$ we may split $\psi(t, \theta)$ into two parts, one due to $r(t)$ and one due to $e(t)$ :

$$
\psi\left(t, \theta_{0}\right)=\psi_{r}\left(t, \theta_{0}\right)+\psi_{e}\left(t, \theta_{0}\right)
$$

where

$$
\begin{aligned}
& \psi_{r}\left(t, \theta_{0}\right)=\frac{1}{C_{0}(q)} \varphi_{r}(t) \\
& \psi_{e}\left(t, \theta_{0}\right)=L^{\prime}\left(q, \theta_{0}\right) C_{0}(q) e(t)+\frac{1}{C_{0}(q)} \varphi_{e}(t)
\end{aligned}
$$

Moreover, since $r(t)$ and $e(t)$ are independent we get

$$
P_{\theta}=\lambda_{0}\left[\bar{E} \psi_{r}\left(t, \theta_{0}\right) \psi_{r}^{T}\left(t, \theta_{0}\right)+\bar{E} \psi_{e}\left(t, \theta_{0}\right) \psi_{e}^{T}\left(t, \theta_{0}\right)\right]^{-1}
$$

With

$$
\bar{R}_{e}=\bar{E} \psi_{e}\left(t, \theta_{0}\right) \psi_{e}^{T}\left(t, \theta_{0}\right)
$$

we thus have, for the direct method,

$$
P_{\theta}^{\mathrm{D}}=\lambda_{0}\left[\bar{E} \frac{1}{C_{0}(q)} \varphi_{r}(t) \frac{1}{C_{0}(q)} \varphi_{r}^{T}(t)+\bar{R}_{e}\right]^{-1}
$$

The key observation here is that $\bar{R}_{e} \geq 0$ regardless of the parameterization of $L(q, \theta)$ (as long as it is sufficiently flexible), so the noise actually helps reducing the variance in the direct method. Furthermore,

$$
P_{\theta}^{\mathrm{D}} \geq \lambda_{0}\left[\bar{E} \frac{1}{C_{0}(q)} \varphi(t) \frac{1}{C_{0}(q)} \varphi^{T}(t)\right]^{-1} \triangleq P_{\theta}^{\mathrm{OD}}
$$

with equality for $L(q, \theta)=L(q)=1 / C_{0}(q)$.

\subsection{The Indirect Method}

If the regulator polynomials $R(q)$ and $S(q)$ are known, one can use the indirect method to get consistent estimates (but not of optimal accuracy) of the open-loop system without having to use a parameter-dependent pre-filter. The idea is to estimate the closed-loop system using a standard (prediction error) method and from this estimate determine the open-loop parameters using the knowledge of the regulator. To circumvent the last step one can parameterize the closed-loop system in terms of the open-loop parameters directly. If both the reference signal $r(t)$ and the input $u(t)$ are measurable one can show that the indirect method with a fixed pre-filter $L(q, \theta)=L_{1}(q)$ is equivalent to the direct method, given that we parameterize the pre-filter as

$$
L(q, \theta)=\frac{L_{1}(q) R(q)}{A(q) R(q)+B(q) S(q)}
$$

The prediction errors are

$$
\varepsilon_{F}(t, \theta)=L(q, \theta)\left(y(t)-\varphi^{T}(t) \theta\right)=L_{1}(q)\left(y(t)-\frac{B(q) S(q)}{A(q) R(q)+B(q) S(q)} r(t)\right)
$$

By straightforward calculations it can be shown that

$$
\psi(t, \theta)=L(q, \theta) \varphi_{r}(t)
$$


where $L(q, \theta)$ is given by (29). At $\theta_{0}$ we have

$$
L\left(q, \theta_{0}\right)=\frac{L_{1}(q) R(q)}{A_{0}(q) R(q)+B_{0}(q) S(q)}
$$

Let

$$
F(q)=L_{1}(q) \frac{C_{0}(q) R(q)}{A_{0}(q) R(q)+B_{0}(q) S(q)}=C_{0}(q) L\left(q, \theta_{0}\right)=\sum_{i=0}^{\infty} f_{i} q^{-i}
$$

and introduce

$$
\tilde{\varphi}_{r}(\tau)=\sum_{i=0}^{\infty} f_{i} \varphi_{r}(\tau+i)
$$

Then $P_{\theta}^{\mathrm{ID}}$, the covariance matrix for the indirect method, is

$$
\begin{aligned}
& P_{\theta}^{\mathrm{ID}}=\lambda_{0}\left[\bar{E} L\left(q, \theta_{0}\right) \varphi_{r}(t) L\left(q, \theta_{0}\right) \varphi_{r}^{T}(t)\right]^{-1}\left[\bar{E} L\left(q, \theta_{0}\right) \tilde{\varphi}_{r}(t) L\left(q, \theta_{0}\right) \tilde{\varphi}_{r}^{T}(t)\right] \\
& \cdot\left[\bar{E} L\left(q, \theta_{0}\right) \varphi_{r}(t) L\left(q, \theta_{0}\right) \varphi_{r}^{T}(t)\right]^{-1}
\end{aligned}
$$

For all choices of the filter $L_{1}(q)$,

$$
P_{\theta}^{\mathrm{ID}} \geq \lambda_{0}\left[\bar{E} \frac{1}{C_{0}(q)} \varphi_{r}(t) \frac{1}{C_{0}(q)} \varphi_{r}^{T}(t)\right]^{-1} \triangleq P_{\theta}^{\mathrm{OID}}
$$

Equality holds if $L_{1}(q)=\left(A_{0}(q) R(q)+B_{0}(q) S(q)\right) /\left(C_{0}(q) R(q)\right)$.

The particular indirect method exemplified here is perhaps the simplest one that can be used. However, the efficiency is not optimal; clearly $P_{\theta}^{\mathrm{OID}} \geq P_{\theta}^{\mathrm{D}}$. With some more effort it is possible to do better: If the closed-loop system is identified using an ARMAX model and the open-loop parameters are solved for using the Markov estimate it can actually be shown that this method is equivalent, as far as the accuracy is concerned, to the direct method. Or, in other words that $P_{\theta}^{\mathrm{ID} \text { ARmax }}=P_{\theta}^{\mathrm{D}_{\text {ARMax }}}$. The details can be found in [6]. See also [10, 2].

\subsection{The Two-stage Method}

The two-stage method [11] is known to be quite robust and give good estimates of the open-loop system. No knowledge of the regulator parameters is required, but the regulator must be linear. This method gives consistent estimates of the open-loop system regardless of the pre-filter used, but the efficiency is sub-optimal.

The idea is to first estimate the noise-free part of the input. Call this estimate $\hat{u}_{r}(t)$. Ideally $\hat{u}_{r}(t)=u_{r}(t)$. Now consider the model

$$
\hat{y}(t \mid \theta)=\frac{B(q)}{A(q)} \hat{u}_{r}(t)
$$

and a pre-filter $L_{1}(q)$. The prediction errors are

$$
\varepsilon_{F}(t, \theta)=L_{1}(q)\left(y(t)-\frac{B(q)}{A(q)} \hat{u}_{r}(t)\right)
$$

With a pre-filter parameterized as $L(q, \theta)=L_{1}(q) / A(q)$ the prediction errors can alternatively be written

$$
\varepsilon_{F}(t, \theta)=L(q, \theta)\left(y(t)-\hat{\varphi}^{T}(t) \theta\right)
$$


with $\theta$ as in (6) and

$$
\hat{\varphi}(t)=\left[-y(t-1), \ldots,-y\left(t-n_{a}\right), \hat{u}_{r}\left(t-n_{k}\right), \ldots, \hat{u}_{r}\left(t-n_{k}-n_{b}\right)\right]^{T}
$$

With

$$
\begin{aligned}
\hat{y}_{r}(t) & =\frac{B(q)}{A(q)} \hat{u}_{r}(t) \\
\hat{\varphi}_{r}(t) & =\left[-\hat{y}_{r}(t-1), \ldots,-\hat{y}_{r}\left(t-n_{a}\right), \hat{u}_{r}\left(t-n_{k}\right), \ldots, \hat{u}_{r}\left(t-n_{k}-n_{b}\right)\right]^{T}
\end{aligned}
$$

the gradient vector $\psi(t, \theta)$ becomes

$$
\psi(t, \theta)=L(q, \theta) \hat{\varphi}_{r}(t)
$$

Suppose that

$$
\varepsilon\left(t, \theta_{0}\right)=L_{1}(q)\left(y(t)-\frac{B_{0}(q)}{A_{0}(q)} \hat{u}_{r}(t)\right)=H(q) \tilde{e}(t)=\sum_{i=0}^{\infty} h_{i} \tilde{e}(t-i)
$$

for some zero mean, white noise signal $\tilde{e}(t)$ with variance $\tilde{\lambda}_{0}$. With

$$
\tilde{\hat{\varphi}}_{r}(\tau)=\sum_{i=0}^{\infty} h_{i} \hat{\varphi}_{r}(\tau+i)
$$

we then have

$$
\begin{aligned}
& P_{\theta}^{\mathrm{T}}=\tilde{\lambda}_{0}\left[\bar{E} L\left(q, \theta_{0}\right) \hat{\varphi}_{r}(t) L\left(q, \theta_{0}\right) \hat{\varphi}_{r}^{T}(t)\right]^{-1}\left[\bar{E} L\left(q, \theta_{0}\right) \tilde{\hat{\varphi}}_{r}(t) L\left(q, \theta_{0}\right) \tilde{\hat{\varphi}}_{r}^{T}(t)\right] \\
& \cdot\left[\bar{E} L\left(q, \theta_{0}\right) \hat{\varphi}_{r}(t) L\left(q, \theta_{0}\right) \hat{\varphi}_{r}^{T}(t)\right]^{-1}
\end{aligned}
$$

Denote the spectrum of a signal $s(t)$ by $\Phi_{s}(\omega)$. Typically, $\Phi_{\hat{u}_{r}}(\omega)<\Phi_{u_{r}}(\omega)$ and $\tilde{\lambda}_{0}>\lambda_{0}$ which implies that the signal-to-noise ratio tend to be worse than for the indirect method. Hence $P_{\theta}^{\mathrm{T}} \geq P_{\theta}^{\mathrm{ID}}$ in general. If $\hat{u}_{r}(t)=u_{r}(t)$ then, e.g., $\tilde{\lambda}_{0}=\lambda_{0}$ and

$$
P_{\theta}^{\mathrm{T}} \geq \lambda_{0}\left[\bar{E} \frac{A_{0}(q) R(q)+B_{0}(q) S(q)}{A_{0}(q) C_{0}(q) R(q)} \varphi_{r}(t) \frac{A_{0}(q) R(q)+B_{0}(q) S(q)}{A_{0}(q) C_{0}(q) R(q)} \varphi_{r}^{T}(t)\right]^{-1} \triangleq P_{\theta}^{\mathrm{OT}}
$$

Equality holds if $L_{1}(q)=\left(A_{0}(q) R(q)+B_{0}(q) S(q)\right) /\left(C_{0}(q) R(q)\right)$.

\section{Instrumental Variable Methods}

\subsection{General}

Consider the linear regression (5). Let $\zeta(t)$ denote an IV vector (of the same size as $\varphi(t)$ ) and $L(q)$ be a pre-filter, then with the standard IV method the parameter estimate is computed as

$$
\hat{\theta}_{N}=\left[\frac{1}{N} \sum_{t=1}^{N} \zeta(t) L(q) \varphi^{T}(t)\right]^{-1} \frac{1}{N} \sum_{t=1}^{N} \zeta(t) L(q) y(t)
$$

given that the indicated inverse exists. Consistency requires the instruments be chosen such that

$$
\bar{E}\left[\zeta(t) L(q) C_{0}(q) e(t)\right]=0
$$

Depending on the choices of instruments $\zeta(t)$ and pre-filter $L(q)$ different instrumental variable "methods" result. 
Suppose that (49) holds. Define

$$
F(q)=L(q) C_{0}(q)=\sum_{i=0}^{\infty} f_{i} q^{-i}
$$

and

$$
\tilde{\zeta}(\tau)=\sum_{i=0}^{\infty} f_{i} \zeta(\tau+i)
$$

Then the covariance matrix $P_{\theta}$ for the IV method is given by $[8,5]$ :

$$
P_{\theta}^{\mathrm{IV}}=\lambda_{0}\left[\bar{E} \zeta(t) L(q) \varphi^{T}(t)\right]^{-1}\left[\bar{E} \tilde{\zeta}(t) \tilde{\zeta}^{T}(t)\right]\left[\bar{E} \zeta(t) L(q) \varphi^{T}(t)\right]^{-T}
$$

For all choices of $\zeta(t)$ and $L(q)$,

$$
P_{\theta}^{\mathrm{IV}} \geq \lambda_{0}\left[\bar{E} \frac{1}{C_{0}(q)} \varphi(t) \frac{1}{C_{0}(q)} \varphi^{T}(t)\right]^{-1} \triangleq P_{\theta}^{\mathrm{OIV}}\left(=P_{\theta}^{\mathrm{OD}}\right)
$$

Equality holds in the last equation if $\zeta(t)=1 / C_{0}(q) \varphi(t)$ and $L(q)=1 / C_{0}(q)$ (given that these choices satisfy (49)). However, this means we need exact knowledge of the true noise polynomial $C_{0}(q)$ and therefore optimal accuracy cannot be achieved in practice.

If the instruments are chosen as filtered versions of $r(t)$ then (49) will be automatically satisfied since $r(t)$ and $e(t)$ are independent. The resulting method will here be denoted RIV ('R' for reference signal). Another possibility is to use delayed versions of the regression vector, $\zeta(t)=$ $\varphi(t-k)$. To satisfy (49), the delay $k$ has to be larger than the maximal delay in $L(q) C_{0}(q)$. This method will be referred to as PIV ('P' for past regressors).

\subsection{RIV}

For this method it can be shown that (see [9]):

$$
P_{\theta}^{\mathrm{RIV}}=\lambda_{0}\left[\bar{E} \zeta(t) L(q) \varphi_{r}^{T}(t)\right]^{-1}\left[\bar{E} \tilde{\zeta}(t) \tilde{\zeta}^{T}(t)\right]\left[\bar{E} \zeta(t) L(q) \varphi_{r}^{T}(t)\right]^{-T}
$$

Moreover, for any pre-filter $L(q)$ and any $\zeta(t)$ constructed from $r(t)$ through filtering we have that

$$
P_{\theta}^{\mathrm{RIV}} \geq \lambda_{0}\left[\bar{E} \frac{1}{C_{0}(q)} \varphi_{r}(t) \frac{1}{C_{0}(q)} \varphi_{r}^{T}(t)\right]^{-1} \triangleq P_{\theta}^{\mathrm{ORIV}}\left(=P_{\theta}^{\mathrm{OID}}\right)
$$

Equality holds if $\zeta(t)=\frac{1}{C_{0}(q)} \varphi_{r}(t)$ and $L(q)=\frac{1}{C_{0}(q)}$.

\subsection{PIV}

For this method, the instruments are chosen to be past regressors. Suppose that

$$
L(q)=\sum_{i=0}^{n_{l}} l_{i} q^{-i}
$$

In order to satisfy the consistency requirement, admissible instruments are $\zeta(t)=\varphi(t-k)$ where $k \geq n_{l}+n_{c}+1$. Introduce

$$
F(q)=L(q) C_{0}(q)=\sum_{i=0}^{n_{l}+n_{c}} f_{i} q^{-i}
$$


and

$$
\tilde{\varphi}(\tau)=\sum_{i=0}^{n_{l}+n_{c}} f_{i} \varphi(\tau+i)
$$

Then

$$
P_{\theta}^{\mathrm{PIV}}=\lambda_{0}\left[\bar{E} \varphi(t-k) L(q) \varphi^{T}(t)\right]^{-1}\left[\bar{E} \tilde{\varphi}(t-k) \tilde{\varphi}^{T}(t-k)\right]\left[\bar{E} \varphi(t-k) L(q) \varphi^{T}(t)\right]^{-T}
$$

This can be better or worse than for the RIV method depending, e.g., on how $L(q)$ and the delay $k$ are chosen. To gain more insight in how $P_{\theta}^{\mathrm{ORIV}}$ and $P_{\theta}^{\mathrm{PIV}}$ compare we will use the following standard result on positive definite matrices [4]: let $X, Y$ and $Z$ be matrices of compatible dimension and also let $Y$ and $Z$ be invertible then

$$
Y^{-1} X Y^{-T} \geq Z^{-1} \Leftrightarrow\left[\begin{array}{cc}
X & Y \\
Y^{T} & Z
\end{array}\right] \geq 0
$$

Therefore, we have

$$
\begin{gathered}
P_{\theta}^{\mathrm{PIV}} \geq P_{\theta}^{\mathrm{ORIV}} \Leftrightarrow \\
\bar{E}\left[\begin{array}{cc}
\tilde{\varphi}(t-k) \tilde{\varphi}^{T}(t-k) & \varphi(t-k) L(q) \varphi^{T}(t) \\
L(q) \varphi(t) \varphi^{T}(t-k) & \frac{1}{C_{0}(q)} \varphi_{r}(t) \frac{1}{C_{0}(q)} \varphi_{r}^{T}(t)
\end{array}\right] \geq 0
\end{gathered}
$$

The left-hand-side of equation (62) equals

$$
\begin{aligned}
\bar{E}\left[\begin{array}{cc}
\tilde{\varphi}(t-k) \tilde{\varphi}^{T}(t-k) & \varphi(t-k) L(q) \varphi^{T}(t) \\
L(q) \varphi(t) \varphi^{T}(t-k) & \frac{1}{C_{0}(q)} \varphi_{r}(t) \frac{1}{C_{0}(q)} \varphi_{r}^{T}(t)
\end{array}\right] \\
=\bar{E}\left[\begin{array}{cc}
\tilde{\varphi}(t-k) \tilde{\varphi}^{T}(t-k) & \tilde{\varphi}(t-k) \frac{1}{C_{0}(q)} \varphi^{T}(t) \\
\frac{1}{C_{0}(q)} \varphi(t) \tilde{\varphi}^{T}(t-k) & \frac{1}{C_{0}(q)} \varphi_{r}(t) \frac{1}{C_{0}(q)} \varphi_{r}^{T}(t)
\end{array}\right] \\
=\bar{E}\left[\begin{array}{cc}
\tilde{\varphi}_{r}(t-k) \tilde{\varphi}_{r}^{T}(t-k) & \tilde{\varphi}_{r}(t-k) \frac{1}{C_{0}(q)} \varphi_{r}^{T}(t) \\
\frac{1}{C_{0}(q)} \varphi_{r}(t) \tilde{\varphi}_{r}^{T}(t-k) & \frac{1}{C_{0}(q)} \varphi_{r}(t) \frac{1}{C_{0}(q)} \varphi_{r}^{T}(t)
\end{array}\right] \\
+\bar{E}\left[\begin{array}{cc}
\tilde{\varphi}_{e}(t-k) \tilde{\varphi}_{e}^{T}(t-k) & \tilde{\varphi}_{e}(t-k) \frac{1}{C_{0}(q)} \varphi_{e}^{T}(t) \\
\frac{1}{C_{0}(q)} \varphi_{e}(t) \tilde{\varphi}_{e}^{T}(t-k) & 0
\end{array}\right]
\end{aligned}
$$

Note that the first matrix in the right-hand-side of equation (63) is positive semi-definite while the second is indefinite. Thus the accuracy for PIV can be better or worse than that of RIV. Equation (63) also tells us when it is likely that PIV gives better accuracy than RIV: when the feedback loop is noisy. Under all circumstances $P_{\theta}^{\mathrm{PIV}} \geq P_{\theta}^{\mathrm{OIV}}=P_{\theta}^{\mathrm{OD}}$.

\section{Discussion}

So far we have reviewed and in some cases extended results for the accuracy of a number of PE and IV methods. We have seen that the direct PE method gives the smallest variance of all methods, which is natural if one think of the relation of this method to the maximum likelihood (ML) method. A key point in understanding the differences between this method and the other $\mathrm{PE}$ methods is to realize that in the direct method the whole input spectrum helps in reducing the variance, whereas in the alternative methods only the noise-free part of the input spectrum contributes. This is natural when you think of the reason why the other methods give consistent estimates despite the correlation between the noise and the input: they neutralize this complicating fact by not using the noisy part of the input! As an example this has the advantage that we may use output error models together with fixed noise models to shape the bias in the PE setting. The drawback is of course that the effective signal-to-noise ratio will be sub-optimal, thus deteriorating 
the results. In a low-noise situation this will not be a problem but with noisy data the difference can be quite dramatic.

The EIV scheme [1] can, to a certain extend, be interpreted as PIV with the pre-filter chosen to be unity ${ }^{1}$. This means that the instruments are delayed regressors, where the delay must exceed the maximum delay in $C_{0}(q)$ for consistency. The quality of any IV estimate depends critically on the correlation between the instruments and the regressors as well as the loss of correlation between the instruments and the noise. The RIV an PIV are two possible choices when the system operates in closed-loop and from (63) we see that, depending on the noise level, either of these methods are preferable. Consider the extreme situation where no reference signal is applied, RIV cannot be used but PIV and EIV may still work and this depends on the identifiability requirements of these two algorithms ${ }^{2}$. Thus for the case where signal-to-noise ratio is low, PIV and EIV are likely to give better estimate than RIV and the indirect method but there is no guarantee that the efficiencies of PIV and EIV are anywhere close to optimal in this case. This erratic type of statistical behavior of PIV and EIV is also shared by some other subspace algorithms, as pointed out in [13], and can be corrected by using say subspace fitting [13]. This discussion also reveals that PIV and EIV — in contrast to the indirect, two-stage and RIV methods - use both the noise-free and noisy parts of the input to reduce the variance. Despite the fact that these two schemes use the noisy part of the input, they do not generally give optimal estimates because the cross-correlation, rather than the auto-correlation, is used to reduce the variance. The special case is when the noise model is known exactly, then one can use the auto-correlation to achieve minimum variance on par with the direct method.

Note that the fact that PIV may give a smaller variance than optimal RIV is in no contradiction to the optimality result of RIV in [9]. This optimality result is based on the assumption that the instruments are generated by filtering the reference input. The instruments used in PIV clearly do not satisfy this assumption.

Altogether this gives new insights into what factors affect the accuracy of different closed-loop identification methods. These issues will now be illustrated in a small example.

\section{Example}

Let the true plant be given by

$$
y(t)+0.5 y(t-1)=u(t-1)+e(t)
$$

and the feedback be

$$
u(t)=r(t)-0.25 y(t)-0.5 y(t-1)
$$

Both $r(t)$ and $e(t)$ are assumed to be white noise sequences with variance unity and $\lambda_{0}$, respectively. Note that for this system $C_{0}(q)=1$. Also, let the pre-filter $L(q)=1$.

\footnotetext{
${ }^{1}$ The reason we use the phrase 'to a certain extend' is that EIV can be interpreted as PIV on the conceptual level but not on the algorithmic level. On the algorithmic level, EIV estimates state space quadruple $(A, B, C, D)$ in two steps: first the $(A, C)$ pair and then follow by $(B, D)$. The first step is conceptually similar to PIV. However, this is not true for second step, as the $(B, D)$-estimation algorithm in [1] generally gives larger variance than that of PIV, though in principle it gives consistent estimate. This can be explained as follows: the algorithm divides the entire data batch into two disjoint halves, the first half is used as instruments while the second half as regressors. For a long data length, it is not too difficult to see, as usual with hindsight, that the instruments are not very correlated with the regressors. A way to rectify this is to use a reference signal whose first half is identical to its second half.

${ }^{2}$ For PIV, equation (59) suggests that controller has to be of sufficiently high order for the plant to be identifiable if no external reference input is applied. For EIV, its persistence of excitation requirement, as in some other subspace schemes, is still an open problem [13]. However, we conjecture that a requirement is that the controller must be of sufficiently high order in addition to others.
} 
By straightforward computations it can be shown that for this system we have

$$
\begin{array}{cc}
R_{r} \triangleq \bar{E} \varphi_{r}(t) \varphi_{r}^{T}(t)=\left[\begin{array}{cc}
\frac{16}{9} & 0 \\
0 & \frac{4}{3}
\end{array}\right] & R_{e} \triangleq \bar{E} \varphi_{e}(t) \varphi_{e}^{T}(t)=\lambda_{0}\left[\begin{array}{cc}
\frac{16}{9} & 0 \\
0 & \frac{1}{3}
\end{array}\right] \\
R_{r, 1} \triangleq \bar{E} \varphi_{r}(t-1) \varphi_{r}^{T}(t)=\left[\begin{array}{cc}
-\frac{8}{9} & \frac{2}{3} \\
-\frac{4}{3} & -\frac{1}{3}
\end{array}\right] & R_{e, 1} \triangleq \bar{E} \varphi_{e}(t-1) \varphi_{e}^{T}(t)=\lambda_{0}\left[\begin{array}{cc}
-\frac{8}{9} & \frac{2}{3} \\
-\frac{1}{3} & -\frac{1}{12}
\end{array}\right] \\
R_{r, 2} \triangleq \bar{E} \varphi_{r}(t-2) \varphi_{r}^{T}(t)=\left[\begin{array}{cc}
-\frac{2}{9} & -\frac{1}{2} \\
\frac{1}{4} & -\frac{5}{12}
\end{array}\right] & R_{e, 2} \triangleq \bar{E} \varphi_{e}(t-2) \varphi_{e}^{T}(t)=\lambda_{0}\left[\begin{array}{cc}
-\frac{2}{9} & -\frac{1}{2} \\
\frac{1}{4} & -\frac{5}{48}
\end{array}\right]
\end{array}
$$

and

$$
\hat{R}_{r} \triangleq \bar{E} \frac{A_{0}(q) R(q)+B_{0}(q) S(q)}{A_{0}(q) C_{0}(q) R(q)} \varphi_{r}(t) \frac{A_{0}(q) R(q)+B_{0}(q) S(q)}{A_{0}(q) C_{0}(q) R(q)} \varphi_{r}^{T}(t)=\left[\begin{array}{cc}
\frac{4}{3} & 0 \\
0 & 1
\end{array}\right]
$$

If we first study the PE methods we see that

$$
\begin{aligned}
& P_{\theta}^{\mathrm{OD}}=\lambda_{0}\left(R_{r}+R_{e}\right)^{-1}=\lambda_{0}\left[\begin{array}{cc}
\frac{9}{16\left(1+\lambda_{0}\right)} & 0 \\
0 & \frac{3}{4+\lambda_{0}}
\end{array}\right] \\
& P_{\theta}^{\mathrm{OID}}=\lambda_{0} R_{r}^{-1}=\lambda_{0}\left[\begin{array}{cc}
\frac{9}{16} & 0 \\
0 & \frac{3}{4}
\end{array}\right] \\
& P_{\theta}^{\mathrm{OT}}=\lambda_{0} \hat{R}_{r}^{-1}=\lambda_{0}\left[\begin{array}{cc}
\frac{3}{4} & 0 \\
0 & 1
\end{array}\right]
\end{aligned}
$$

Clearly, $P_{\theta}^{\mathrm{OD}} \leq P_{\theta}^{\mathrm{OID}}$ for all $\lambda_{0}$, and the difference is larger the larger $\lambda_{0}$ is. Thus the direct method gives better accuracy than the indirect method. We also have that $P_{\theta}^{\mathrm{OID}}<P_{\theta}^{\mathrm{OT}}$ which implies that the two-stage method gives the worst accuracy of the PE methods.

For the IV methods we first note that $P_{\theta}^{\mathrm{OD}}=P_{\theta}^{\mathrm{OIV}}$ and $P_{\theta}^{\mathrm{OID}}=P_{\theta}^{\mathrm{ORIV}}$. Hence $P_{\theta}^{\mathrm{OIV}} \leq P_{\theta}^{\mathrm{ORIV}}$ for all $\lambda_{0}$. We stress that the optimal instruments (OIV) are rarely admissible, although this is the case here. For PIV we have that

$$
P_{\theta}^{\mathrm{PIV}}(k)=\lambda_{0}\left(R_{r, k}+R_{e, k}\right)^{-1}\left(R_{r}+R_{e}\right)\left(R_{r, k}+R_{e, k}\right)^{-T}
$$

It can be shown that $P_{\theta}^{\mathrm{PIV}}(k), k=1,2$, is smaller than $P_{\theta}^{\mathrm{ORIV}}$ when $\lambda_{0}$ is large, but that the situation is reversed when $\lambda_{0}$ is small. By explicitly evaluating the difference $P_{\theta}^{\mathrm{PIV}}(k)-P_{\theta}^{\mathrm{OIV}}$ for a number of noise levels, see Table 1, we find that this difference changes from being negative definite to positive definite as the noise level increases. For this particular example, we remark that $k=0$ is also admissible and this results in $P_{\theta}^{\mathrm{PIV}}(0)=P_{\theta}^{\mathrm{OIV}}=P_{\theta}^{\mathrm{OD}}$, which is the best possible variance that can be achieved.

Finally, we examine two extreme situations: when $\lambda_{0}$ is zero and when it becomes infinity. The former situation corresponds to noise free data and all methods give optimal estimate. The later situation corresponds to the case where no reference signal is applied. On one hand, we have both $P_{\theta}^{\mathrm{OID}}$ and $P_{\theta}^{\mathrm{OT}}$ become infinity. On the other hand both $P_{\theta}^{\mathrm{PIV}}$ and $P_{\theta}^{\mathrm{OIV}}$ converge to constant matrices and they are

$$
\begin{aligned}
P_{\theta, \infty}^{\mathrm{OD}} & =\left[\begin{array}{cc}
\frac{9}{16} & 0 \\
0 & 3
\end{array}\right] \\
P_{\theta, \infty}^{\mathrm{PIV}}(1) & =\left[\begin{array}{ll}
\frac{117}{64} & \frac{27}{16} \\
\frac{27}{16} & \frac{21}{4}
\end{array}\right] \\
P_{\theta, \infty}^{\mathrm{PIV}}(2) & =\left[\begin{array}{ll}
\frac{1197}{256} & \frac{27}{64} \\
\frac{27}{64} & \frac{93}{16}
\end{array}\right]
\end{aligned}
$$


Table 1: Summary of variance results

\begin{tabular}{|c|c|c|c|c|c|}
\hline$\lambda_{0}$ & 0.01 & 0.1 & 1 & 10 & 100 \\
\hline$P_{\theta}^{\mathrm{OD}}-P_{\theta}^{\mathrm{OID}}$ & neg. def. & neg. def. & neg. def. & neg. def. & neg. def. \\
\hline$P_{\theta}^{\mathrm{OID}}-P_{\theta}^{\mathrm{OT}}$ & neg. def. & neg. def. & neg. def. & neg. def. & neg. def. \\
\hline \multicolumn{6}{|c|}{$P_{\theta}^{\mathrm{OD}}=P_{\theta}^{\mathrm{OIV}} \quad$ and } \\
\hline$P_{\theta}^{\mathrm{OIV}}-P_{\theta}^{\mathrm{PIV}}(1)$ & neg. def. & neg. def. & neg. def. & neg. def. & neg. def. \\
\hline$P_{\theta}^{\mathrm{PIV}}(1)-P_{\theta}^{\mathrm{ORIV}}$ & pos. def. & pos. def. & pos. def. & indef. & neg. def. \\
\hline$P_{\theta}^{\mathrm{OIV}}-P_{\theta}^{\mathrm{PIV}}(2)$ & neg. def. & neg. def. & neg. def. & neg. def. & neg. def. \\
\hline$P_{\theta}^{\mathrm{PIV}}(2)-P_{\theta}^{\mathrm{ORIV}}$ & pos. def. & pos. def. & indef. & indef. & neg. def. \\
\hline
\end{tabular}

\section{Conclusions}

We have performed a detailed comparison study on the accuracy of a number of identification methods that can be applied when the system operates in closed-loop. These methods include those that are based on PE method as well as those that are based on IV method. The general conclusion is this: a closed-loop identification algorithm must make use of the auto-correlation of both the noise-free and noisy parts of the data, coupled with a correct noise model, in order to give optimal accuracy.

A directly applied PE method gives optimal accuracy under reasonable conditions. Other PE methods, such as the indirect method and the two-stage method, typically give worse accuracy than the direct method because only the noise-free part of the data is used.

With optimally chosen instruments the accuracy of IV method coincides with that of the direct method. However, this requires exact knowledge of the noise model and therefore cannot be achieved in practice. Two alternative choices of instruments have been suggested: one is to use filtered versions of the reference signal as instruments, another is to use delayed regressors. The former choice does not give optimal estimate again because it uses only the noise-free part of the data. The latter choice can also be seen to correspond to using the EIV subspace method presented in [1]. This identification method does make use of both the noise-free and noisy parts of the data but its accuracy is sub-optimal partly because cross-correlation, rather than auto-correlation, is used to reduce the variance. It was also argued that the former choice of instruments is likely to give more accurate estimates than the latter when the signal-to-noise ratio is high, but the opposite is true when the noise-level is high.

\section{References}

[1] C.T. Chou and M. Verhaegen. Subspace algorithm for the identification of multivariable dynamic errors-in-variables models. Automatica, 33(10):1857-1869, October 1997.

[2] U. Forssell and L. Ljung. Closed-loop Identification Revisited. Technical Report LiTH-ISY-R1959, Department of Electrical Engineering, Linköping University, Linköping, Sweden, 1997.

[3] I. Gustavsson, L. Ljung, and T. Söderström. Identification of processes in closed loop identifiability and accuracy aspects. Automatica, 13:59-75, 1997.

[4] R.A. Horn and C.R. Johnson. Matrix Analysis. Cambridge University Press, 1985.

[5] L. Ljung. System Identification: Theory for the User. Prentice-Hall, Inc., Englewood Cliffs, New Jersey, 1987.

[6] T. Söderström, L. Ljung, and I. Gustavsson. On The Accuracy Of Identification And the Design Of Identification Experiments. Technical Report 7428, Department of Automatic Control, Lund Institute of Technology, Lund, Sweden, 1974. 
[7] T. Söderström and P. Stoica. Instrumental Variable Methods for System Identification. Springer-Verlag, 1983.

[8] T. Söderström and P. Stoica. System Identification. Prentice Hall, 1989.

[9] T. Söderström, P. Stoica, and E. Trulsson. Instrumental variable methods for closed-loop systems. In Proc. 10th IFAC World Congress, pages 364-369, 1987.

[10] T. Söderström, P. Stoica, and B. Friedlander. An Indirect Prediction Error Method for System Identification. Automatica, 27:183-188, 1991.

[11] P. M. J. Van den Hof and R. J. P. Schrama. An Indirect Method for Transfer Function Estimation from Closed Loop Data. Automatica, 29(6):1523-1527, 1993.

[12] P. M. J. Van den Hof and R. J. P. Schrama. Identification and Control - Closed-loop Issues. Automatica, 31(12):1751-1770, 1995.

[13] M. Viberg, B. Wahlberg, and B. Ottersten. Analysis of state space system identification methods based on instrumental variables and subspace fitting. Automatica, 33(9):1603-1617, 1997. 